\section{La farmacoeconomía en la industria farmacéutica y el sistema sanitario de Cuba}

\author{
Manuel M. Collazo Herrera ${ }^{1}$ \\ y Norma Casademunt Balbín ${ }^{2}$
}

Durante las dos últimas décadas la evaluación económica de tecnologías y programas de salud ha registrado un auge espectacular en el escenario internacional. En el caso de los medicamentos, tal evaluación contribuye a armonizar las necesidades de la población con los recursos económicos disponibles. De hecho, la selección de una opción terapéutica considerando simultáneamente sus costos y beneficios permite adoptar decisiones a partir de criterios más racionales, objetivos y transparentes (1-3).

En Cuba, a raíz de la importancia que las autoridades sanitarias otorgan a la farmacoeconomía, la industria farmacéutica ha empezado a interesarse por dichas técnicas analíticas y a utilizarlas para desarrollar sus estrategias de investigación y desarrollo (IyD), producción y comercialización.

Por esta razón, dentro de los estudios dirigidos a un aprovechamiento farmacológico más racional y eficiente, la farmacoeconomía ocupa un lugar muy especial en tanto permite determinar las líneas de investigación de medicamentos sobre la base de su eficacia clínica comprobada y su conveniencia económica.

\section{La eficiencia de los tratamientos farmacológicos}

El drástico aumento de los costos de muchas tecnologías sanitarias en todo el mundo y las crecientes presiones por recortar los presupuestos de salud en la mayoría de los países han llevado a plantear la necesidad de una evaluación económica de las intervenciones de salud (4). En tal sentido, la farmacoeconomía podría definirse como la aplicación del análisis económico al campo de los medicamentos (5) o, si se quiere, como la aplicación de la teoría económica a la farmacoterapia (6).

La evaluación económica de medicamentos se engloba dentro de una disciplina más amplia - la evaluación económica de tecnologías sanitariascuya finalidad es escoger aquellas opciones que tengan un impacto más positivo en la salud (7). Dado que toda evaluación se basa en la comparación, la evaluación económica de medicamentos podría definirse como la determinación de la eficiencia (relación entre costos y efectos) de un tratamiento farmacológico y su comparación con la de otras opciones, con el propósito de seleccionar la que presente una relación costo a efecto más favorable (8). 
Desde una perspectiva clínica, la utilización de una tecnología se justifica si su efectividad (la consecución de un objetivo en condiciones reales) o, por lo menos, su eficacia (la consecución de un objetivo en condiciones ideales) es positiva. Sin embargo, desde la perspectiva económica el acento se pone en la eficiencia (9). Pensar en términos de eficiencia implica considerar la efectividad de un proceso en relación con los recursos que requiere. La razón es obvia. Dado que los recursos son limitados respecto a sus posibles aplicaciones, cuanto menos recursos sean necesarios para conseguir un determinado objetivo, tanto mejor, ya que así quedarán más recursos disponibles para lograr otros propósitos en términos de salud (10).

En consecuencia, la evaluación económica consiste en determinar los efectos que se derivarían de seguir cada una de las opciones o subsiguientes cursos de acción posibles en una situación de elección, y comparar dichas opciones en términos de su eficiencia social, o sea, su contribución a mejorar el bienestar de las personas en la sociedad (11). En teoría este proceso se refleja en los estudios de evaluación económica en los que, para determinar el bienestar, es preciso tener en cuenta los costos y los beneficios sociales, es decir, todos los aspectos que se derivan de una intervención sanitaria dada (12).

Además, la evaluación económica de los medicamentos permite ponderar sistemática y explícitamente toda la información disponible para hacer determinada elección, incluidos los juicios de valor, inevitables en cualquier proceso racional de toma de decisiones (13).

Las técnicas de análisis de la evaluación farmacoeconómica corresponden habitualmente a una de las cuatro categorías siguientes: análisis de minimización de costos (AMC), análisis de costo-beneficio (ACB), análisis de costo-efectividad (ACE) y análisis de costo-utilidad (ACU). Tales categorías se diferencian entre sí por la forma en que miden los efectos sobre la salud de las opciones evaluadas (14).

La evaluación económica de medicamentos constituye un instrumento de trabajo útil para lograr un aprovechamiento óptimo de los recursos humanos, materiales y financieros que se destinan a los servicios de salud (15). Su aplicación práctica se basa en el principio de que el paciente debe recibir la atención que requiere para mantener su salud y está orientada al desarrollo de estudios que permitan seguir ese principio mediante la eficiencia y racionalidad en el uso de los fármacos (16).

\section{Aplicaciones prácticas de la farmacoeconomía}

Las aplicaciones prácticas de los estudios farmacoeconómicos pueden ser útiles en multitud de situaciones relacionadas con la toma de decisiones (17), por ejemplo:

- Decisiones en materia de IyD de una empresa farmacéutica. La evaluación económica de la lista de productos en desarrollo permite valorar su potencial económico, las posibilidades de obtener buenos resultados y la conveniencia o no de continuar trabajando en un producto determinado.

- Decisiones de fijación de precios. Los estudios farmacoeconómicos pueden indicar si las ventajas socioeconómicas de un nuevo fármaco justifican su financiación, o contribuir a la negociación de su precio.

- Decisiones sobre formularios. La determinación de los efectos de los fármacos en la práctica diaria mediante la comparación con nuevas opciones, análisis prospectivos y retrospectivos, y la realización de modelos económicos pueden resultar claves en la inclusión de un producto en formularios.

- Recomendaciones sobre la decisión clínica. Los protocolos clínicos deben tener en cuenta no solo la eficacia y la efectividad de las opciones terapéuticas, sino también su relación costo-efectividad.

- Estudios de vigilancia poscomercialización. Los estudios farmacoeconómicos mantienen constantemente actualizados los registros de los efectos de los fármacos en la práctica clínica real, lo que permite reevaluar su eficiencia e informar sobre posibles modificaciones en el precio, formularios o recomendaciones terapéuticas.

En la actualidad, dado el elevado costo que implica desarrollar un producto nuevo, las empresas farmacéuticas se preocupan por proporcionar fármacos cada vez más eficientes, eficaces y mejor tolerados, lo cual se traduce en un claro avance dentro del acervo terapéutico disponible (18).

Con esta idea la industria farmacéutica, cada vez más asiduamente, diseña y realiza análisis de evaluación económica durante todo el ciclo de vida de los medicamentos, desde los primeros estadios de su desarrollo hasta su lanzamiento al mercado (19). La evaluación económica permite alcanzar diferentes objetivos en el campo de la farmacoterapia (20), incluidos los siguientes:

- Racionalizar el uso de los medicamentos, controlar los resultados del tratamiento elegido y los efectos adversos, y garantizar la disponibilidad constante de fármacos de calidad a costos razonables.

- Elaborar una lista básica de medicamentos adecuados a las necesidades del país, para guía y referencia de los servicios de salud y sistemas de información, que esté avalada mediante la evaluación técnico-económica correspondiente. 
- Desarrollar esquemas terapéuticos adecuados a las enfermedades prevalecientes en cada país, que hagan posible establecer una lista de prioridades por enfermedades, teniendo en cuenta la efectividad de los medicamentos.

- Diseños de sistemas de suministros de medicamentos compatibles con las diferentes estructuras del sector de la salud y con la disponibilidad de recursos económicos, así como con los procedimientos administrativos del país.

- Verificar durante todo el proceso de IyD, producción y comercialización de un nuevo fármaco que dicho producto tendrá aceptación en el mercado.

En las sociedades industrializadas que son capaces de regular y controlar los costos de producción de bienes de salud y donde la cobertura sanitaria es prácticamente universal, la farmacoeconomía podrá sin duda ayudar a mejorar la toma de decisiones clínicas, sobre todo cuando se debe escoger entre varios tratamientos similares. Todo ejercicio, sin embargo, debe estar dirigido no solo a controlar costos, sino también a mejorar la eficiencia para alcanzar la equidad en el acceso a los servicios de salud (21).

En los países en desarrollo, en cambio, donde una parte importante de la población carece de acceso a medicamentos y donde muchas personas mueren a causa de enfermedades para las cuales existen medicamentos preventivos, la farmacoeconomía tendrá enfoques y características muy diferentes. Más que la contención de costos, lo que se buscará es racionalizar los recursos existentes y conseguir fuentes suplementarias de financiamiento, de tal forma que se garantice la extensión de la cobertura a toda la población (4). Una farmacoeconomía de este tipo estaría más centrada en la selección de productos y sería también un elemento más para promover lo que la Organización Mundial de la Salud ha denominado "uso racional de los medicamentos" (4).

\section{La incorporación de la farmacoeconomía en Cuba}

El Ministerio de Salud Pública de Cuba ha trazado una estrategia destinada a incrementar la eficiencia y la calidad de los servicios de salud, y a garantizar la sostenibilidad del sistema, especialmente en términos financieros (22). Puede decirse sin dudas que el sistema de salud cubano es eficiente cuando se toman en cuenta su alcance territorial y social, su desarrollo tecnológico y su carácter gratuito y universal. Ningún sistema que no tuviera cierto grado de eficiencia habría sido capaz de subsistir y mantener todas las características se- ñaladas, en un período de crisis tan profunda como la sufrida por la economía nacional. No obstante, nunca se debe desechar la idea del ahorro y el uso racional de los recursos que el estado asigna a este sistema que, al igual que la educación, no es un negocio sino un deber social (23).

Hay en el sistema sanitario cubano un grado determinado de eficiencia que puede verse en los indicadores de salud -y otros logros - alcanzados y mantenidos incluso en el marco de una situación económica muy desfavorable (23). Claro está que esto no quiere decir, en modo alguno, que se haya conseguido toda la eficiencia necesaria y menos aún la deseada. No se ha conseguido todavía en razón de la insuficiencia de recursos que tiene el sistema y por no haberse adoptado medidas que podrían resolver este problema, como por ejemplo, la evaluación del costo de las tecnologías sanitarias y la armonización entre la eficiencia técnica y la económica.

Debido a que Cuba, aun cuando es un país del Tercer Mundo, cuenta con un sistema sanitario comparable a los de los países desarrollados, la farmacoeconomía en esta nación tendrá un doble propósito: racionalizar el uso de los medicamentos seleccionados y mejorar los niveles de eficiencia de los tratamientos.

La incorporación de estas técnicas de análisis por parte de la industria farmacéutica y del sistema nacional de salud representa una vía muy importante para lograr que la evaluación económica comience a incorporarse en toda la esfera de la circulación de los medicamentos, incluidas la selección, adquisición, distribución y prescripción (24).

En este sentido, el Centro de Investigación y Desarrollo de Medicamentos (CIDEM), perteneciente al Ministerio de Salud Pública, ha realizado las primeras evaluaciones económicas de tratamientos farmacológicos para hipertensión arterial, insuficiencia cardíaca y cardiopatía isquémica, úlceras pépticas, infecciones vaginales, diabetes, asma, control de la glucosa en orina y administración de insulina, prevención de las sepsis postoperatorias con antibióticos, quimioterapia del cáncer, neumonía nosocomial en cuidados intensivos, intervenciones alimentario-nutrimentales en pacientes operados de cáncer de colon y otras enfermedades. Estos estudios mantienen actualizados los efectos de los fármacos en la práctica clínica habitual, lo que permite evaluar en términos económicos su eficiencia y su efectividad terapéutica.

Con estos estudios farmacoeconómicos se posibilita elaborar y poner en práctica una política nacional para el uso racional de los medicamentos, que se ajuste a la situación económico-financiera que atraviesa el país, así como mejorar la eficiencia de los tratamientos. De esta forma se conjugan los 
intereses de la industria farmacéutica con los de la prestación de servicios de salud, permitiendo a ambos sectores trazar proyecciones estratégicas de trabajo conjuntas que den lugar a una mejor utilización de los fármacos con los recursos existentes.

En cuanto a la industria farmacéutica, el proceso de investigación de nuevos fármacos tiene como requisito fundamental, además de las estimaciones económico-financieras del proyecto, realizar una evaluación farmacoeconómica que demuestre las ventajas socioeconómicas del medicamento que se desea obtener, en relación con el problema de salud que se pretende solucionar y con respecto a otro medicamento que se busca sustituir.

Las empresas farmacéuticas planifican su producción sobre la base de los criterios farmacoepidemiológicos y farmacoeconómicos del MINSAP, aspectos estos que determinan y rigen el proceso de selección del fármaco, a la vez que indican los volúmenes de producción necesarios para satisfacer la demanda nacional.

Dentro de este proceso de búsqueda de eficiencia y racionalidad de los recursos para nuestro sistema de salud, la preparación docente desempeña un papel importante. El CIDEM organiza cursos de farmacoeconomía que se ofrecen anualmente en el marco de su Programa de Superación Profesional, a los cuales asisten profesionales de distintas especialidades como médicos, farmacéuticos, economistas y licenciados en enfermería. Hasta la fecha se han ofrecido cinco cursos de 40 horas de duración, con valor de cuatro créditos académicos avalados por el Centro Nacional de Superación Técnico Profesional de la Salud (CENAPET) y una participación de más de 160 especialistas. Asimismo, se han organizado distintos eventos científicos nacionales e internacionales sobre la economía de la salud en el país.

\section{Conclusión}

Es necesario que los profesionales que trabajan en el área de los medicamentos promuevan la idea de que la eficiencia económica debe llevar implícita la elección de alternativas en la prestación de los servicios, de forma tal que se consiga el mayor benefi- cio posible con los recursos puestos a disposición del sistema de salud. Esto quiere decir que dicha selección deberá estar basada en la relación existente entre la efectividad terapéutica y el costo del tratamiento. Hace falta entonces continuar promoviendo el interés por la farmacoeconomía e impulsar nuevos análisis que conlleven metodologías y técnicas más actualizadas, a fin de disponer de todas las experiencias acumuladas y ampliar el número de evaluaciones económicas de medicamentos para su aplicación práctica en todo el sistema nacional de salud. Esta labor redundará en una mejor utilización de los fármacos, principalmente en la prescripción.

Todavía queda mucho por hacer para lograr eficiencia y racionalidad suficientes en el uso de los medicamentos: evaluar los distintos programas nacionales de salud, los proyectos de investigación y las diversas tecnologías sanitarias. A la farmacoeconomía le ha correspondido una función importante en la consecución de estos objetivos de trabajo para nuestro sistema de salud. Ahora es indispensable incorporar estos conocimientos con el propósito de que estimulen y respalden un movimiento innovador de conceptos y estilos de trabajo dentro del campo sanitario.

\section{SYNOPSIS}

\section{Pharmacoeconomics in the pharmaceutical industry and health system of Cuba}

This piece describes how pharmacoeconomic studies promote rational and efficient drug use. The pharmaceutical industry of Cuba has become more interested in such analytical techniques and has begun to use them to develop strategies for research and development, production, and product marketing. This paper describes research related to pharmacological treatments, through practical examples of pharmacoeconomic evaluations carried out jointly by the pharmaceutical sector and the health care system in Cuba. These efforts demonstrate the applicability that pharmacoeconomics has for health decision-making based on clinical effectiveness and the costs of the pharmacotherapeutics used.

\section{REFERENCIAS}

1. Eisemberg JM, Glick H, Koffer H. Pharmacoeconomics: economic evaluation of pharmaceuticals. En: Strom B, ed Pharmacoepidemiology. Nueva York: Churchill Livingston, 1989 .

2. McGhan WF, Lemus JW. Guidelines for pharmacoeconomic studies. Clin Ther 1992;14:486-494.
3. Drummond $\mathrm{M}$, et al. Economic evaluation of pharmaceuticals: a European perspective. Pharmacoeconomics 1993;4:173-186.

4. Velázquez G. Farmacoeconomía: ¿evaluación científica o estrategia comercial? Rev Panam Salud Publica 1999; 5(1):54- 57.

5. SOIKOS. Glosario de términos y conceptos de uso frecuente en la evaluación económica de medicamentos y programas sanitarios. Barcelona: Química Farmacéutica Bayer; 1996

6. Milne R. [Editorial] Pharmacoeconomics 1992;1(1):1.

7. Panerai R, Mohr J. Evaluación de tecnologías en salud: metodologías para países en desarrollo. Washington, D.C.: 
Organización Panamericana de la Salud; 1990.

8. Sacristán J, Badia X, Rovira J. Farmacoeconomía: evaluación económica de medicamentos. Madrid : Editores Médicos; 1995.

9. Badia X, Rovira J. Evaluación económica de medicamentos: un instrumento para la toma de decisión práctica clínica y política sanitaria. Barcelona: Luzàn 5; 1994

10. Luce BR, Elixhauser A. Standards for the socioeconomics evaluation of health care services. Berlin: Springer-Verlag; 1990.

11. Drummond MF, Stoddart GL, Torrance GW. Methods for the economic evaluation of health care programmes. Toronto: Oxford Medical Publications; 1987.

12. Drummond MF. Issues in the conduct of economic evaluations of pharmaceutical products. Pharmacoeconomics 1994; 6(5):405-411.

13. Drummond $M$, Stoddart G, Torrance G. Métodos para la evaluación económica de los programas de atención de la salud. Madrid: Editorial Díaz de Santos; 1990.

14. Bootman JL, Towsend RJ, Mc Ghan WF. Principles of pharmacoeconomics. Cincinnati: Harvey Whitney; 1991.

15. Collazo M, Flores N. Farmacoeconomía: la eficiencia en la evaluación de los tratamientos farmacológicos. Rev Cubana Farmacia 2000;34(1):63-69.

16. Collazo M, Rovira J. Criterios sobre la evaluación económica de los medicamentos antineoplásicos. Rev Cubana Oncol 1997;13(1):23-30.

17. Canadian Coordinating Office for Health Technology. Guidelines for economic evaluation of pharmaceutics. Canadá: CCOHT; 1994.

18. Soto J. Evaluación económica de medicamentos: perspectiva de la industria farmacéutica en nuestro país. Rev Atenc Farm 1999;1(6):600-608.

19. Andersson F. Why is the pharmaceutical industry investing increasing amounts in health economic evaluations? Int J Tech Assess Health Care 1995;11:750-761.
20. Rovira J, Collazo M. La evaluación económica, ¿debe ser de interés para los oncólogos? III Jornadas Canarias de Oncología. Madrid: Arán Ediciones; 1998.

21. Evans D. The changing healthcare environment. Pharmacoeconomics 1992;1(1): 5-7.

22. Cosme J, Cárdenas J, Miyar R. Economía y salud: conceptos, retos y estrategias. Washington, D.C.: Organización Panamericana de la Salud; 1999. Serie Desarrollo de la Representación OPS/OMS Cuba No. 22.

23. Cárdenas J, Cosme J. Eficiencia en la atención primaria de salud. Washington, D.C.: Organización Panamericana de la Salud; 2000. Serie Desarrollo de la Representación OPS/OMS Cuba. No. 24.

24. Dumoulin J, Kaddar M, Velázquez G. Guía de análisis económico del circuito del medicamento. Ginebra: Organización Mundial de la Salud; 1995. WHO/ DAP/95.2.

While the successful execution of a longitudinal study is a difficult task and "ideal" studies in a free-living population are, for practical purposes, usually impossible, less than "perfect" studies may nevertheless be valuable as a basis for implementing community health activities. In the course of investigating a population, an increased public awareness of health problems occurs which, in itself, is valuable from a health education standpoint.

[Realizar estudios longitudinales con buenos resultados es una tarea difícil, y un estudio "ideal" en una población abierta es por lo general, tarea imposible. No obstante, se pueden efectuar estudios - no tan perfectos- como base para promover actividades de salud comunitaria. Al investigar una población, el público adquiere mayor conciencia de los problemas de salud, lo cual es valioso en sí mismo desde el punto de vista de la educación en materia de salud.]

Thomas R. Dawber, William B. Kannel y Lorna P. Lyell An approach to longitudinal studies in a community: the Framingham Study Annals New York Academy of Sciences 1963; 107:539-556 